\title{
A new family of magnetic adhesion based wall- climbing robots
}

\author{
Stefano Seriani ${ }^{1}$, Lorenzo Scalera ${ }^{2}$, Alessandro Gasparetto ${ }^{2}$ and Paolo \\ Gallina $^{1}$ \\ ${ }^{1}$ University of Trieste, Italy,e-mail: sseriani@units.it; pgallina@units.it \\ ${ }^{2}$ University of Udine, Italy, e-mail: alessandro.gasparetto@uniud.it; \\ scalera.lorenzo@spes.uniud.it
}

\begin{abstract}
This paper is devoted to climbing robots that adhere to the wall through permanent magnetic elements. If the surface on which they adhere is not ferromagnetic, it is necessary for the system to be composed of two subsystems (master and follower carts), arranged in a sandwich configuration, with the surface to climb interposed between the two. However, this configuration does not allow the robot to descend from the wall where it is climbing and to move freely on the floor (because of the presence of the follower). This paper shows how to remove this limitation. In fact, the system is able to automatically detach the follower when the robot has to move on the floor and recover it when it has to climb.
\end{abstract}

Keywords: Climbing robots, magnetic adhesion.

\section{Introduction}

Nowadays, the application of mobile robots is becoming more and more important in several different fields: exploration of harzardous environments [1,2], inspection and surveillance, [2-4] materials handling in the industrial and commercial field [5]. In this context, a special class of mobile robot is that of robots capable of climbing on wall surfaces. Among these, there are many studies in literature about climbing robots that adhere to the wall through permanent magnetic elements [613].

Permanent magnets have several advantages with respect to other adhesion systems: mainly low cost and no energy required for adhesion, since no suction cups or vacuum generation systems are required [6].

They work very well when the surfaces to which they must adhere are ferromagnetic. The permanent magnet can be housed into the robotic system. Alternatively, the wheels themselves can be made of magnetic material [11]. In the first case, it the presence of an air gap must be taken into account, thus the force of adhesion is 
reduced. In the second case, the adhesion force is higher because the wheels are in direct contact with the ferromagnetic surface. But, at the same time, the contact is on a line.

In some cases, the robot can even climb without the presence of wheels, always taking advantage of the principle of magnetic adhesion, like for the crawler-type robots [9].

These types of robots are used in various applications, for example to perform non-destructive tests $[7,8]$ or to inspect the hulls of ships $[10,11]$.

If the surface on which they adhere is non-ferromagnetic, it is necessary for the system to be composed of two subsystems, in the following indicated as master cart and follower cart. They are arranged in a sandwich configuration, with the surface to climb interposed between the two. A simple mockup of the master/follower robot is shown in Fig. 1. The robot is in upside down position, hanging from a cardboard ceiling.

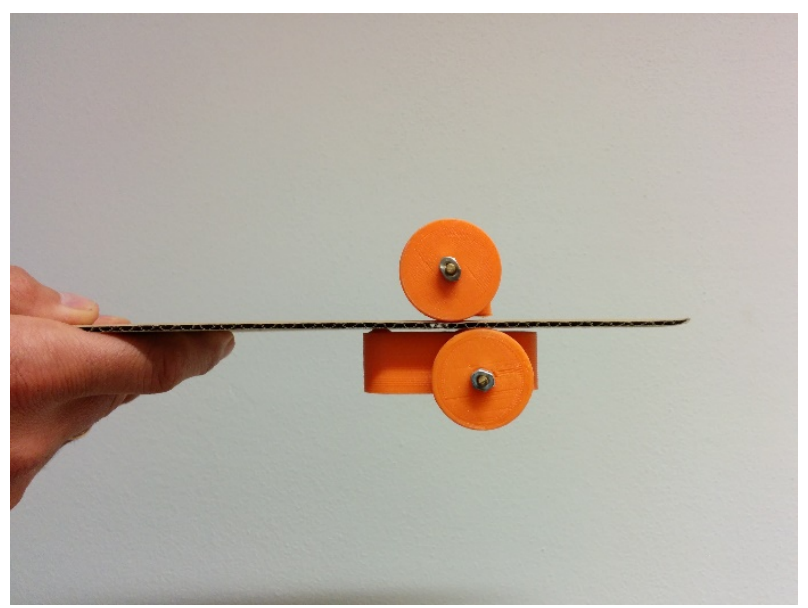

Fig. 1. Master/follower robot configuration. The robot

The master is the active robot. In general, it is provided with controlled wheels and with the other tools required to perform a given task: cameras and sensors for inspections; actuators and manipulators to operate on the environment.

The slave is provided with passive wheels, to reduce the friction on the surface to climb. It just houses ferromagnetic or permanent magnetic elements. Its purpose is to close the magnetic field flow lines generated by the master.

These kind of robots can climb on thin plastic surfaces, glasses or tissues.

One main drawback of the master-follower configuration is that the robot can not descend from the wall where it is climbing and move freely on the floor (because of the presence of the follower). This paper shows how remove this limitation. In fact, the system is able to automatically detach the follower when the robot has to move on the floor and recover it when it has to climb. 
When the master moves on the floor, the follower is stored in a position close to the climb ramp (See Fig. 2, a)). As the master reaches the ramp, the follower links to the master, thus providing the adhesion force to climb (See Fig. 2 b)). The most critical situation is when the master steps off the ramp and leaves the follower in its storage position.

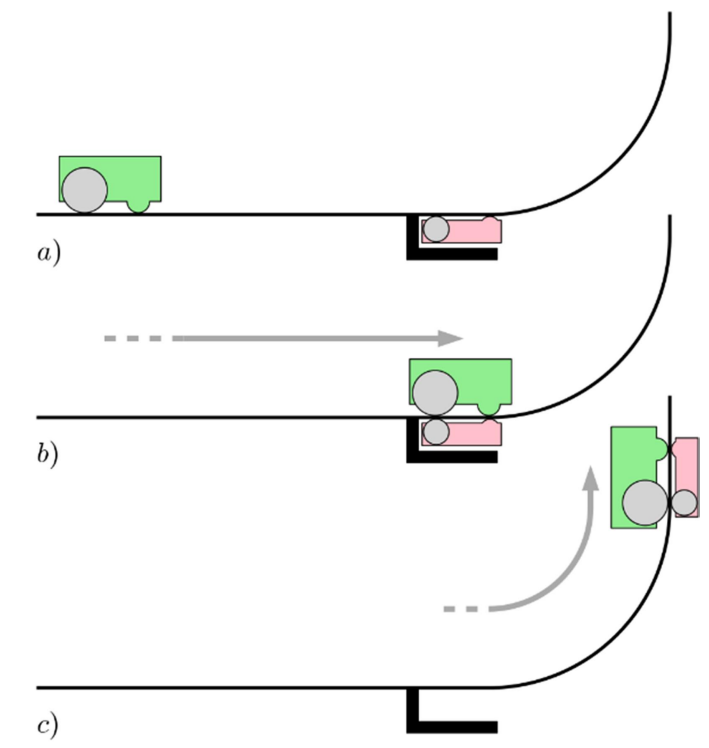

Fig 2. The three phase of the transition from floor motion to climbing motion. a) the master moves on the floor and the follower is stored under the ramp; $b$ ) the follower links to the master; c) master and follower climbs the surface.

In order for the master to be able to detach itself from the follower, the torque produced by its wheels must be sufficiently high to exceed the adhesion force produced by the magnets. In addition, the friction force between the surface and the wheels must be enough to prevent sliding. These requirements have been expressed into mathematical relationships, essential for the choice of the magnets, for the choice of the wheel materials and for the design of the robot.

The paper is organized as follows. In Section 2, some mathematical relationships and conditions are introduced. Section 3 shows how to use the theory for the robot design. Eventually conclusions are given in Section 4.

\section{Robot model and theory}

The pair of master and follower carts is placed on opposite sides of a magnetically permeable sheet of material of negligible thickness. Referring to Fig. 3, a), we consider master and follower cart wheels of radius $r_{r}$ and $r_{f}$ and centered in 
points $\Omega_{r}$ and $\Omega_{f}$ respectively; each cart is equipped with a magnet positioned at points $H_{r}$ and $H_{f}$ respectively. We can define the distance $h_{r}=r_{r}-\left\|\Omega_{r}-H_{r}\right\|$, and, similarly, $h_{f}$. The magnets exchange an attractive force which is modeled as $F_{m}=k / d^{2}$, where $k$ is an experimental parameter describing the dipole moments associated with the magnets. This model introduces a certain uncertainty because it does not consider misalignments between the dipole vectors. However, we find that for a preliminary investigation, it can be adopted successfully, especially considering that the misalignment is small. The distance $d$ between the two magnets can be written as follows,

$d=\frac{h_{r}+r_{f}-\left(r_{f}-h_{f}\right) \operatorname{co}}{\cos }=\frac{h_{r}+r_{f}}{\cos \alpha}-\left(r_{f}-h_{f}\right)$

The wheel of the master is able to produce the traction force $F_{a}=\tau / r_{r} \leq$ $F_{\mu, \text { lim }}=\mu\left(F_{m, \perp}+m_{r} g\right)$, where $\tau$ is the wheel torque, $\mu$ is the static friction coefficient between wheel and ground, $F_{m, \perp}=F_{m} \cos \alpha, m_{r}$ is the mass of the master, and $g$ is gravity acceleration.

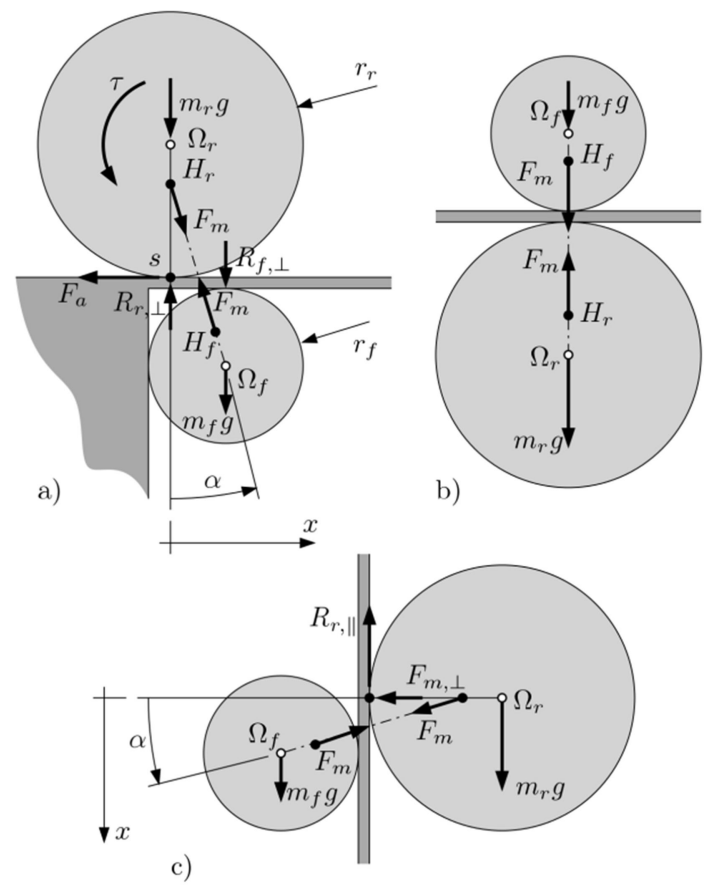

Fig. 3 Model diagram for the system in different configurations; in a), the regular orientation is shown during parking, in b) the up-side down configuration is shown, whereas in c) a configuration where the robot is climbing a vertical wall. 
As Fig. 3, a) shows, we can define the angle $\alpha$ as that between the virtual vertical plane (i.e. orthogonal to the x-axis) and the virtual plane that contains the axles of the wheels of the master and follower cart. As can be seen, since $d=f(\alpha)$, we also have that $F_{m}, F_{\mu, \text { lim }}$ and, in general, all forces which depend on the magnetic interaction are dependent from $\alpha$.

We can define at least three cases which are notable in studying this system. Based on the specific case, we should first define some quantities; in Fig. 3, a), the ground exerts normal reaction forces $R_{r, \perp}$ and $R_{f, \perp}$ to the robot and the follower respectively, whereas in Fig. 3, c), we can define the value $R_{r, \|}$ that represents the reaction of the driving surface to the forces acting on the robot (namely weight and magnetic force). The cases are as follows:

i. Up-side up orientation (Fig. 3, a)), with a stopper for the follower robot, relative to the parking operation of the follower cart; in this case, the follower robot is prevented from advancing by an obstacle. The aim is to allow the master to free itself of the follower, by overcoming the magnetic attraction and driving away. In order to keep traction the following must hold: $F_{a} \leq F_{\text {lim }, \mu}$ (Condition 1),

ii. Up-side down orientation (Fig. 3, b)), when the robot is overturned by $180^{\circ}$; in order to keep the robot adherent to the driving surface, the following must hold: $F_{m}>m_{r} g$ (Condition 2),

iii. Vertical wall climb (Fig. 3, c)); the first condition in this case is: $R_{r, \|}<$ $F_{\mu, l i m}=\mu F_{m, \perp}($ Condition 3). The second condition is related to the follower cart magnetic attraction overcoming its weight: $F_{m, \|}>m_{f} g$ (Condition 4).

Compliance to all conditions is required for the system to be completely functional.

\section{Design considerations related to a real scenario}

A pseudo-static numerical computation of the problem was performed based on the behavior of the magnetic force $F_{m}$ with regards to the misalignment $x_{\Omega_{f}}$ between the wheels. The main geometrical relation is that of Eq. 1. The step of the simulation was $\Delta x_{\Omega_{f}} \cong 0.3 \mathrm{~mm}$.

We implemented the values of the prototype in the model; $r_{r}=r_{f}=$ $0.0165 \mathrm{~m}, h_{f}=h_{r}=0.007 \mathrm{~m}, \mu=0.8, m_{r}=0.041 \mathrm{Kg}, m_{f}=0.034 \mathrm{Kg}$. In Fig. 4 the results are reported for the first two cases, i. and ii.

The plot of Fig. 4, a) shows the torque required to keep the robot in equilibrium for different values of $x_{\Omega_{f}}$, where $x_{\Omega_{f}}$ ( $x$ coordinate of the point $\Omega_{f}$ ) represents the misaligning between the master and the slave along the surface. There is a univo- 
cal relationship between $\alpha$ and $x_{\Omega_{f}}$. As expected, the torque starts at zero (magnets are aligned), and subsequently presents a peak followed by a decline that reflects the weakening of the magnetic interaction.

In Fig. $4, b$ ) the result of the configuration $i$. is shown, describing the parking operation of the follower cart. The allowed $F_{\text {lim, },}$ is shown, along with the effective value of the required traction force $F_{a}$, both charted against $x_{\Omega_{f}}(\alpha)$. It is critical that $F_{a} \leq F_{\text {lim }, \mu}$ (Condition 1) for the robot to keep traction against the driving surface; it can be seen that in this case, the condition is satisfied $\forall x_{\Omega_{f}}(\alpha)$.

In Fig. $4, c)$, the vertical force $F_{m, \|}$ is charted along with the weight $m_{f} g$ of the follower cart; it can be seen that the system satisfies Condition 4 for $0.007<$ $x_{\Omega_{f}}<0.024$.
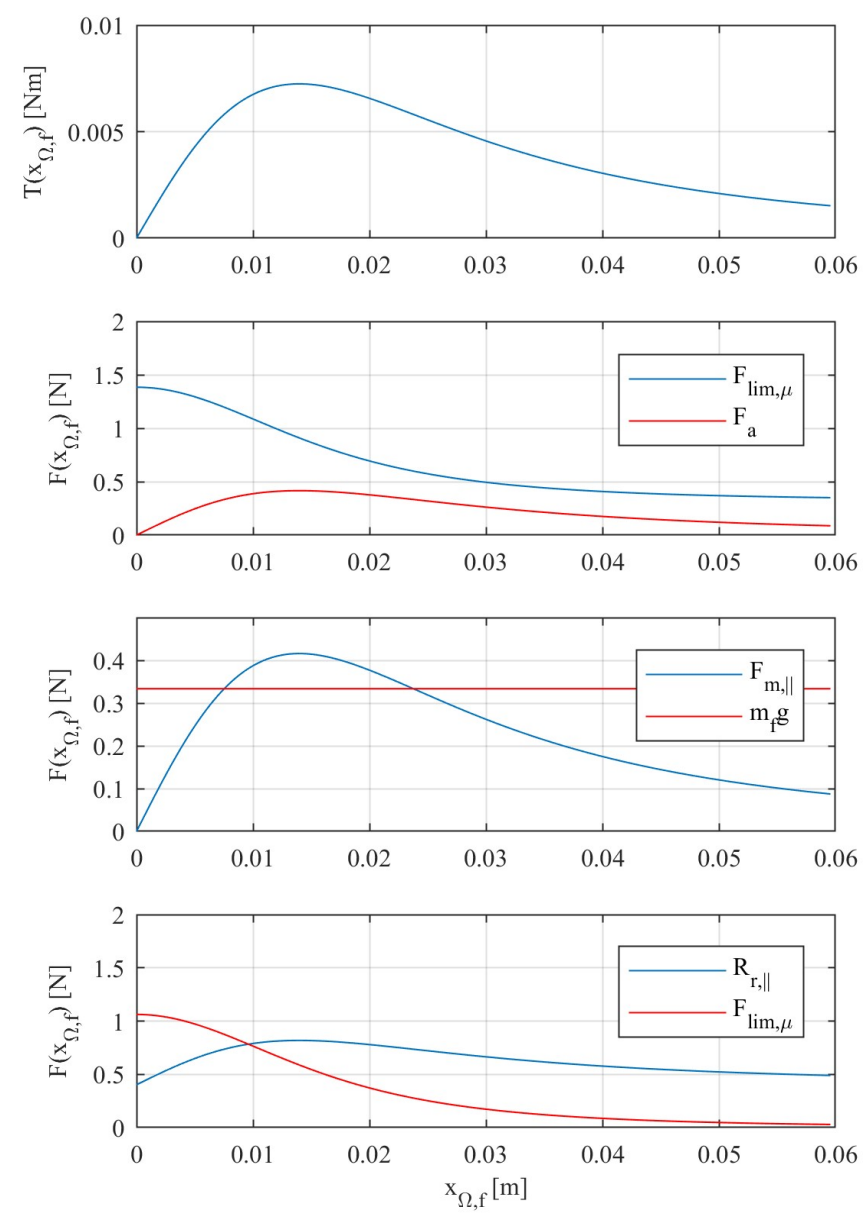
Fig. 4. Results for the simulations for values $x_{\Omega_{f}}(\alpha) \in[0,0.14]$. In a) the torque $T\left(x_{\Omega_{f}}\right)$ is shown in case $i$; in b) the comparison is shown between the traction force $F_{a}$ at contact point and the friction limit $F_{\text {lim, } \mu}$ in case $i$; in c), related to case iii, the comparison is reported between the magnetic force $F_{m}$ tangential component $F_{m, \|}$ and the weight of the follower cart $m_{f} g$; in d), the value for $R_{r, \|}$ is reported against the friction limit $F_{\text {lim, } \mu}$, in case iii.

Finally, in Fig. 4, d), it can be seen that Condition 3 can be satisfied; indeed $R_{r, \|} \leq F_{\text {lim, } \mu} \forall x_{\Omega_{f}}: x_{\Omega_{f}}<0.010$, approximately. Condition 2 can always be satisfied, since $F_{m}>m_{r} g$, being $F_{m} \cong-1.33 N$ and $m_{r} g \cong 0.4 N$.

Moreover, from the plot in Fig. 4 , c), we can see that two points of equilibrium exist where $F_{m, \|}=m_{f} g$; the left-most one is a stable equilibrium point, whereas the right-most one is instable. This (Cond. 4), coupled to the admissible values given by Cond. 3, 2 and 1, gives a feasibility envelope of $0.007<x_{\Omega_{f}}<0.010$; in fact, if the value $x_{\Omega_{f}}$ is inside the envelope, all conditions are met and the system is functional in cases $\mathrm{i}$, ii and iii.

\section{Conclusions}

This paper presented a magnetic based climbing robot composed of a master and a follower capable to move on a floor (without the follower) and climbing on a nonferromagnetic surface (linking to the follower). A proper design to take into consideration all the mechanical parameters involved in the model of the robot has been presented. In particular, 4 conditions have been derived and verified for a real case.

\section{References}

1. S. Seriani, P. Gallina, A. Wedler, "Dynamics of a Tethered Rover on Rough Terrain", 1st International Conference of IFToMM Italy, IFIT 2016, Advances in Italian Mechanism Science, pp. 355-361, (2016)

2. S. Seriani, P. Gallina, A. Wedler, "A Modular Cable Robot for Inspection and Light Manipulation on Celestial Bodies", Acta Astronautica, vol. 123, pp. 145-153, (2016)

3. Bietresato, M., Carabin, G., D'Auria, D., Gallo, R., Ristorto, G., Mazzetto, F., ... \& Scalera, L. (2016, August). A tracked mobile robotic lab for monitoring the plants volume and health. In Mechatronic and Embedded Systems and Applications (MESA), 2016 12th IEEE/ASME International Conference on (pp. 1-6). IEEE.

4. Vidoni, R., Gallo, R., Ristorto, G., Carabin, G., Mazzetto, F., Scalera, L., \& Gasparetto, A. (2017, November). ByeLab: An Agricultural Mobile Robot Prototype for Proximal Sensing and Precision Farming. In ASME 2017 International Mechanical Engineering Congress and Exposition (pp. V04AT05A057-V04AT05A057). American Society of Mechanical Engineers. 
5. Cesetti, A., Scotti, C. P., Di Buo, G., \& Longhi, S. (2010, June). A service oriented architecture supporting an autonomous mobile robot for industrial applications. In Control \& Automation (MED), 2010 18th Mediterranean Conference on (pp. 604-609). IEEE.

6. Cai, J. et al.: The design of permanent-magnetic wheeled wall-climbing robot. IEEE International Conference on Information and Automation, ICIA 2017, art. no. 8078979, 604608 (2017)

7. Faruq Howlader, M.O. and Sattar, T.P.: Development of magnetic adhesion based climbing robot for non-destructive testing. 7th Computer Science and Electronic Engineering Conference, CEEC 2015 - Conference Proceedings, art. no. 7332708, 105-110 (2015)

8. Faruq Howlader, M.O. and Sattar, T.P.: Design and optimization of permanent magnet based adhesion module for robots climbing on reinforced concrete surfaces Studies in Computational Intelligence, 650, 153-171 (2016)

9. $\mathrm{Hu}, \mathrm{S}$., et al.: Structural design and magnetic force analysis of a new crawler-type permanent magnetic adsorption wall - Climbing. IEEE International Conference on Information and Automation, ICIA 2017, art. no. 8078978, 598-603 (2017)

10. Huang, H. et al.: Design and performance analysis of a tracked wall-climbing robot for ship inspection in shipbuilding. Ocean Engineering, 131, 224-230 (2017)

11. Ishihara, H.: Basic study on wall climbing root with magnetic passive wheels. IEEE International Conference on Mechatronics and Automation, ICMA 2017, art. no. 8016119, 1964-1969 (2017)

12. Mao, J., He, K., Li, J. and Sun, X.: Simulation and experimental verification of permanent magnet adsorption unit for wall-climbing robot. IEEE International Conference on Information and Automation, IEEE ICIA 2016, art. no. 7832000, 1189-1194 (2017)

13. Milella, A., Maglietta, R., Caccia, M. and Bruzzone, G.: Robotic inspection of ship hull surfaces using a magnetic crawler and a monocular camera Sensor Review, 37 (4), 425$435(2017)$ 